\section{PREDICTING FEEDING PROBLEMS IN INFANTS WITH CENTRAL GREY MATTER DAMAGE FOLLOWING HYPOXIC-ISCHAEMIC ENCEPHALOPATHY (HIE)}

M. Martinez-Biarge ${ }^{1,2}$, J. Diez-Sebastian ${ }^{3}$, S. Lawrence ${ }^{4}$, A. Aloysius ${ }^{4}$, M.A. Rutherford ${ }^{5}$, F.M. Cowan ${ }^{1}$

${ }^{1}$ Paediatrics and Imaging Sciences, Imperial College London, London, UK, ${ }^{2}$ Neonatology, ${ }^{3}$ Statistics, La Paz University Hospital, Madrid, Spain, ${ }^{4}$ Speech and Language Therapy, Queen Charlotte and Chelsea-Hammersmith Hospital. Imperial College, London, ${ }^{5}$ Robert Steiner MR

Unit, Imperial College London, London, UK

Background and aims: Feeding problems are common following HIE and are a huge burden for infants and families. We aimed to evaluate, in term newborns with HIE and basal ganglia/thalamic (BGT) lesions, the relation between feeding difficulties and early MRI findings.

Methods: From 1993-2007, all term infants with evidence of perinatal asphyxia, HIE and BGT injury seen on MRI within 6 postnatal weeks were included. BGT, white matter, internal capsule, cortex and brainstem lesions were classified by severity. Cerebral palsy (CP) severity was determined from the Gross Motor Function Classification System (GMFCS).

Results: 175 infants fulfilled the entry criteria. 49 died (23 neonatally, 26 in infancy). Feeding problems occurred in all who died, both early and late, and in $65 \%$ of survivors; $44 \%$ had difficulty with liquids, $48 \%$ with solids and $27 \%$ only tolerated pureed foods. $33 \%$ had a gastrostomy (median age 11 (range 3-48) months). All but 3 of the 82 children with feeding problems had CP, 91\% GMFCS level IV/V. In a logistic regression model BGT/mesencephalic injury was associated with feeding problems $(p<$ 0.001 ) and BGT/pons injury with gastrostomy ( $p<$ 0.001 ). The need for gastrostomy occurred in only $3.5 \%$ of infants with mild/moderate BGT + normal pons but in $90.6 \%$ of surviving infants with severe BGT+ pons injury.

Conclusions: The occurrence and severity of feeding problems in neonates with HIE and BGT injury relates to the severity of the BGT/brainstem lesions. These findings enable planning of care and a better approach to nutrition in these children.
171

\section{DIETARY SUPPLEMENTATION WITH ARGINYL- GLUTAMINE (ARG-GLN) DIPEPTIDE OR \\ DOCOSAHEXAENOIC ACID (DHA) MODULATE HYPEROXIA-INDUCED BRAIN INURY IN NEONATAL MICE}

\author{
X. Liu' ${ }^{1,2}$, N. Li ${ }^{1}$, L. Ma1 ${ }^{1}$, K. Morris ${ }^{3}$, L. Shaw ${ }^{4}$, \\ S. Li Calzi ${ }^{4}$, M.B. Grant ${ }^{4}$, X. Cai ${ }^{1,2}$, J. Neu ${ }^{1}$ \\ ${ }^{1}$ Department of Pediatrics, University of Florida, \\ Gainesville, FL, USA, ${ }^{2}$ Sheng Jing Hospital, \\ Shenyang Liaoning, China, ${ }^{3}$ Global Discovery, \\ Mead Johnson Nutrition Company, Evansville, IN, \\ ${ }^{4}$ Department of Pharmacology and Therapeutics, \\ University of Florida, Gainesville, FL, USA
}

Aims: Using a model of hyperoxia-induced brain injury, we evaluated the potential for Arg-GIn or DHA to counteract the impact of hyperoxia in the developing brain.

Methods: 7-day old pups and their nursing dams were placed in $75 \%$ oxygen for five days. Pups were returned to room air on postnatal day $12\left(\mathrm{P}_{12}\right)$ and supplemented by oral gavage twice daily with ArgGln $(5 \mathrm{~g} / \mathrm{kg} / \mathrm{d})$, DHA $(5 \mathrm{~g} / \mathrm{kg} / \mathrm{d})$ or vehicle through $\mathrm{P}_{17}$. A group of mice kept in room air served as normoxia controls. On $\mathrm{P}_{17}$, pups were sacrificed and brains collected. Caspase- 3 activity was used as a marker of apoptosis. Inflammatory cytokines IL-6 and KC were determined by ELISA. LDH activity served as an index of tissue injury.

Results: Arg-GIn reduced caspase-3 activity by $19 \%$ vs. hyperoxia $(p<0.05)$, while DHA was without effect. Exposure to hyperoxia increased LDH activity by $22 \%$ over normoxia controls. Importantly, the hyperoxia-induced increase in LDH activity was attenuated with Arg-GIn or DHA, by $22 \%$ and $36 \%$, respectively $(p<0.05)$. Although there was a modest induction of IL- 6 by hyperoxia, supplementation with Arg-GIn or DHA completely reversed this effect resulting in IL-6 levels that were $19 \%$ and $30 \%$, respectively, lower than the hyperoxic-vehicle group $(p<0.05)$. DHA significantly reduced $K C$ levels by $34 \%(p<0.05)$ vs. hyperoxic-vehicle, while Arg-GIn had no effect.

Conclusion: These results demonstrate dietary supplementation with Arg-GIn or DHA modulate markers of hyperoxia-induced inflammation and tissue injury, suggesting these nutrients to be of particular importance to the developing brain exposed to supplemental oxygen. 